\title{
A Distributed Middleware Infrastructure for Personalized Services*
}

\author{
Marios D. Dikaiakos \\ Dept. of Computer Science \\ University of Cyprus \\ PO Box 20537, Nicosia, Cyprus \\ mdd@ucy.ac.cy
}

\author{
Demetris Zeinalipour-Yazti \\ Dept. of Computer Science \\ University of California \\ Riverside, CA, USA \\ csyiazti@cs.ucr.edu
}

\begin{abstract}
In this paper we present an overview of eRACE, a modular and distributed intermediary infrastructure that collects information from heterogeneous Internet sources according to registered profiles or end-user requests. Collected information is stored for filtering, transformation, aggregation, and subsequent personalized or wide-area dissemination on the wireline or wireless Internet. We study the architecture and implementation of the main module of eRACE, an HTTP proxy named WebRACE. WebRACE consists of a high-performance, distributed and multithreaded Web crawler, a multithreaded filtering processor and an object cache. We discuss the implementation of WebRACE in Java, describe a number of performance optimizations, and present its performance assessment.
\end{abstract}

\section{Introduction}

The rapid expansion of the Web, and the developments in mobile-device and wirelessInternet technologies have resulted to a large heterogeneity of client devices currently used for accessing Internet services. Furthermore, they have raised the capacity mismatch between clients and Internet servers. To cope with these trends, software infrastructures for

${ }^{*}$ Work supported in part by the grant PENEK 23/2000 from the Research Promotion Foundation of Cyprus. 
Internet services have to: (i) Support seamless access from a variety devices; (ii) Customize content according to the requirements and limitations of different terminal devices. (iii) Support both synchronous (on-demand) and asynchronous modes of interaction with users, thus coping with frequent disconnections of wireless access and user mobility. (iv) Optimize the amount of useful content that reaches users through client devices with limited resources and restricted interfaces, by enabling service personalization, localization and filtering of information. (v) Guarantee high availability and robustness, as well as incremental performance and capacity scalability with an expanding user base.

Possible approaches for coping with these requirements are the so-called end-to-end solutions, where origin servers adapt their content on-the-fly, taking into account consolidated user profiles [35] or the terminal device and network connection involved in a user session [28]. For an end-to-end approach to work properly, however, adaptation software has to be inserted at each origin server. Consequently, software updates have to be propagated to all origin servers whenever new terminal devices and content-encoding protocols emerge. Moreover, on-the-fly adaptation of content can be very time-consuming, leading to a deterioration of user experience.

An alternative approach for providing personalization, content customization, ubiquity and mobility, is the employment of proxies with a functionality significantly extended over what is found in traditional proxies for the wireline [45] or wireless [25] Web. Given the high-performance requirements for high throughput, $24 \times 7$ availability, and performance scalability of next-generation Internet services [10, 33], however, centralized proxies are expected to face performance problems as well, thus necessitating the distribution of their computation, storage and complexity into the networking infrastructure [28, 9]. In such a case, a number of distributed, programmable and possibly mobile intermediary servers would be deployed throughout the network. These servers would mediate between primary information sources and various client systems, providing performance scalability, better sharing of resources, higher cost efficiency and a streamlining of new service provision [9].

The focus of our work is on the development of eRACE, an intermediary infrastructure with enhanced functionality and distributed architecture, to support the development and deployment of personalized services and the provision of ubiquitous access thereof. In this paper we present the design principles and architecture of eRACE. Furthermore, we 
describe the design, implementation and performance assessment of WebRACE, which is an eRACE-proxy dealing with collecting, filtering and caching content from the World-Wide Web, according to personal and service profiles. WebRACE consists of a multithreaded crawler, a multithreaded filtering engine, and an object cache. The remaining of this paper is organized as follows: Section 2 presents an overview of the eRACE architecture. Section 3 describes the main challenges behind WebRACE design and implementation. Sections 4, 5 and 6 describe the main components of WebRACE: the Mini-crawler, the Object Cache and the Annotation Engine. Section 7 presents our experimentation and performance assessment of WebRACE. Furthermore, it presents the performance enhancements that we achieve by caching to cache the crawling state in the Object Cache, and by distributing the crawler to a network of workstations. Section 8 presents an overview of recent related work. We provide our conclusions in Section 9.

\section{The Architecture of eRACE}

\subsection{Overview and Goals}

The extensible Retrieval, Annotation and Caching Engine (eRACE) is a middleware infrastructure designed to support the development and deployment of intermediaries on Internet. Intermediaries are "software programs or agents that meaningfully transform information as it flows from one computer to another" [6,31], and represent a useful abstraction for describing and developing personalized proxies, mobile services, etc.

eRACE is a modular, programmable and distributed proxy infrastructure that collects information from heterogeneous Internet sources and protocols according to eRACE profiles registered within the infrastructure, and end-user requests. Collected information is stored in a software cache for further processing, personalized dissemination to subscribed users, and wide-area dissemination on the wireline or wireless Internet.

eRACE supports personalization by enabling the registration, maintenance and management of personal profiles representing the interests of individual users. Furthermore, its structure allows the easy customization of service provision according to parameters, such as information-access modes (pull or push), client-proxy communication (wireline or wireless; email, HTTP, WAP), and client-device capabilities (PC, PDA, mobile phone, thin 
clients). Ubiquitous service-provision is supported by eRACE thanks to the decoupling of information retrieval, storage and filtering, from content publishing and distribution. The eRACE infrastructure can also easily incorporate mechanisms for providing subscribed users with differentiated service-levels at the middleware level. Finally, the design of eRACE is tuned for providing performance scalability, which is an important consideration given the expanding numbers of WWW users, the huge increase of information sources available on the Web, and the need to provide robust services.

Key design and implementation decisions made to accomplish these goals are described below:

1. The information architecture of eRACE is defined in terms of metadata that represent user account and connection information, user and service profiles, state information of eRACE modules and information exchanges taking place between them. Specifications are defined as XML Document Type Definitions (DTDs) [8]. We chose XML because it is simple, self-descriptive, and extensible. Hence, we can easily extend our descriptions to incorporate new services, terminal devices, and QoS policies. Furthermore, we can re-use existing modules and API's that process XML data. Central to this set of meta-data is the "eRACE profile" and the "eRACE annotation." The eRACE profile is a concise XML description of the operations and transformations that eRACE modules are expected to perform upon heterogeneous Internet sources: information gathering, filtering and caching of retrieved content, transcoding, dissemination, etc. The eRACE-profile DTD is general and expressive so as to represent: (i) personal interests of subscribers, thus supporting the deployment of personalized services over the Web; (ii) generic services that can be deployed on wide-area networks, such as notification systems, portals, mobile services, etc. The results of profile-driven, filtering operations performed upon gathered content are encoded in XML and named eRACE Annotations or Annotation Cache Information (ACI's).

2. Data sharing between modules of the eRACE infrastructure is done through messages that transport XML-encoded information and events. Therefore, we can easily decouple and isolate modules from each other and distribute them physically across machine boundaries at configuration or run-time. Furthermore, modules with stringent performance requirements are multithreaded and employ distributed data-structures [22], to 
allow the exploitation of parallel execution on shared-memory multiprocessor systems and networks of workstations.

3. eRACE translates user requests and eRACE profiles into "eRACE requests," encoded in XML and tagged with QoS information. These requests are scheduled for execution by an eRACE scheduler, which can implement different scheduling policies based on QoS tags. The explicit maintenance of XML-encoded information regarding pending requests and content scheduled for dissemination, makes it easy to keep track of the system's run-time behavior, to compare alternative scheduling algorithms, to implement load-balancing techniques for sustaining high-availability during high loads, and to apply QoS policies with different service levels.

4. eRACE is implemented with Java [21]. Java was chosen for a variety of reasons. Its object-oriented design enhances the software development process, supports rapid prototyping and enables the re-use and easy integration of existing modules. Java class libraries provide support for key features of eRACE: platform independence, multithreading, network programming, high-level programming of distributed applications, string processing, code mobility, compression, etc. Our choice of Java, however, came with a certain risk-factor that arose from known performance problems of this platform and its run-time environment. Performance and robustness are issues of critical importance for systems like eRACE, which must perform as a server, run continuously and sustain high-loads at short periods of time.

5. Support for mobility and disconnected operations of Proxy and Content-Distribution agents will be provided by Mitsubishi's Concordia Mobile Agent platform [29, 30]. For that matter, we have conducted a number of studies to assess the performance and robustness of this platform, with encouraging results [16, 40]. Furthermore, we implemented two earlier prototypes prototype of eRACE $[15,14,46]$ with Concordia; mobile agents were used to implement the communication protocol between users and eRACE servers. To this end, the front-end interface of eRACE was implemented as a Java applet with an embedded transporter able to launch and receive Concordia Agents. "Agent proxies" were implemented as stationary Concordia Agents able launching mobile agents to access and combine information sources over the network. 


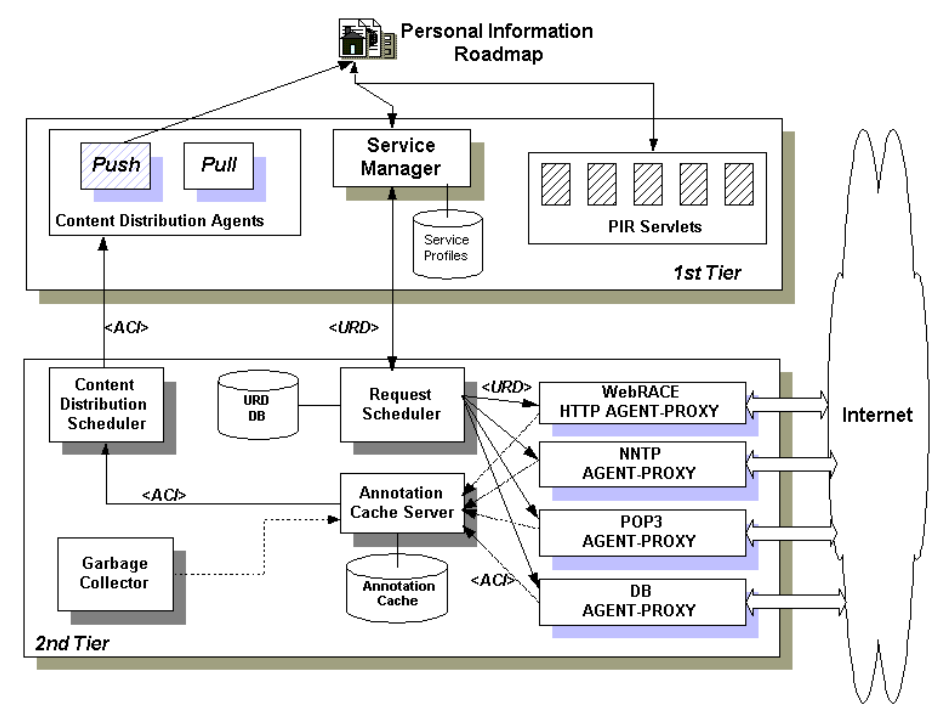

Figure 1: eRACE System Architecture.

\subsection{System Architecture}

\subsubsection{First Tier}

eRACE is organized as a two-tier architecture (see Figure 1). The first tier includes modules that manage services provided to users: the Service Manager, Content-Distribution Agents, and Personal Information Roadmap (PIR) Servlets.

The Service Manager is comprised of modules for handling user connection (authentication, login) and profile management. Each time a user connects to eRACE, the Service Manager notifies other modules so that content starts "flowing" to the user. Furthermore, the Service Manager maintains and manages user and service profiles that are defined and stored as XML data. A profile is a set of long-term, continuously evaluated queries [43]; in eRACE, these queries can be typical queries to Web databases, HTTP requests for WorldWide Web resources, access to general-purpose Search Engines or Subject Cataloging Sites, subscription to Usenet News, etc. Each profile is annotated with a number of data and control parameters. Data parameters are query arguments (e.g., a stock symbol of interest), whereas control parameters determine the frequency of query execution, the expected amount of information gathered from queries (e.g., summary vs. full results), the priority of notification for a given query, etc. Profiles are managed by the Service Manager through the Java API of PDOM [26], which is a persistent Document Object Model. The Service 
Manager translates these profiles into eRACE requests that are forwarded for execution to the second tier of eRACE (see Figure 1).

Content-Distribution Agents retrieve content pertinent to user and service profiles, and maintained in the caches of eRACE's Agent Proxies (with meta-information kept in the Annotation Cache). Content is aggregated by the CDA's, which decide when and how to disseminate it to end-users, following possible terminal- or connection-specific transformations (adaptation, transcoding, etc.). Additional optimizations, such as caching content within the Content-Distribution Agents "near" the PIR Servlets, will be addressed in future work.

eRACE provides users with seamless access to their content through the Personal Information Roadmap (PIR). This is a customized user-interface that implements a simple e-mail based information provision paradigm, seeking to cope with problems of network disorientation and information overloading. The PIR provides a user with a personalized and unified view of her personal information space across different devices. The PIR is implemented with a set of Java Servlets, which transcode it to a format appropriate for the users' terminal connection (HTML for connections over HTTP and WML for connections over WAP). The PIR can be used simultaneously with other tools employed to access information on Internet (browsers, e-mailers, etc).

\subsubsection{Second Tier}

The second tier of eRACE consists of a number of protocol-specific Agent-Proxies like WebRACE, mailRACE, newsRACE and dbRACE that retrieve and cache information from the WWW, POP3 email-accounts, USENET NNTP-news, and Web-database queries respectively (see Figure 1).

Agent-Proxies are driven by a Request Scheduler, which scans continuously a database of Unified Resource Descriptions (URD-DB in Figure 1) and schedules URD's for execution to the corresponding Agent-Proxy. A Unified Resource Description (URD) is an XML-encoded data structure, which is part of an "eRACE request," and describes source information, processing directives and urgency information for Internet sources monitored by eRACE. In essence, each URD represents a request to retrieve and process content from a particular Internet source on behalf of a particular user or service. A typical URD request is shown 


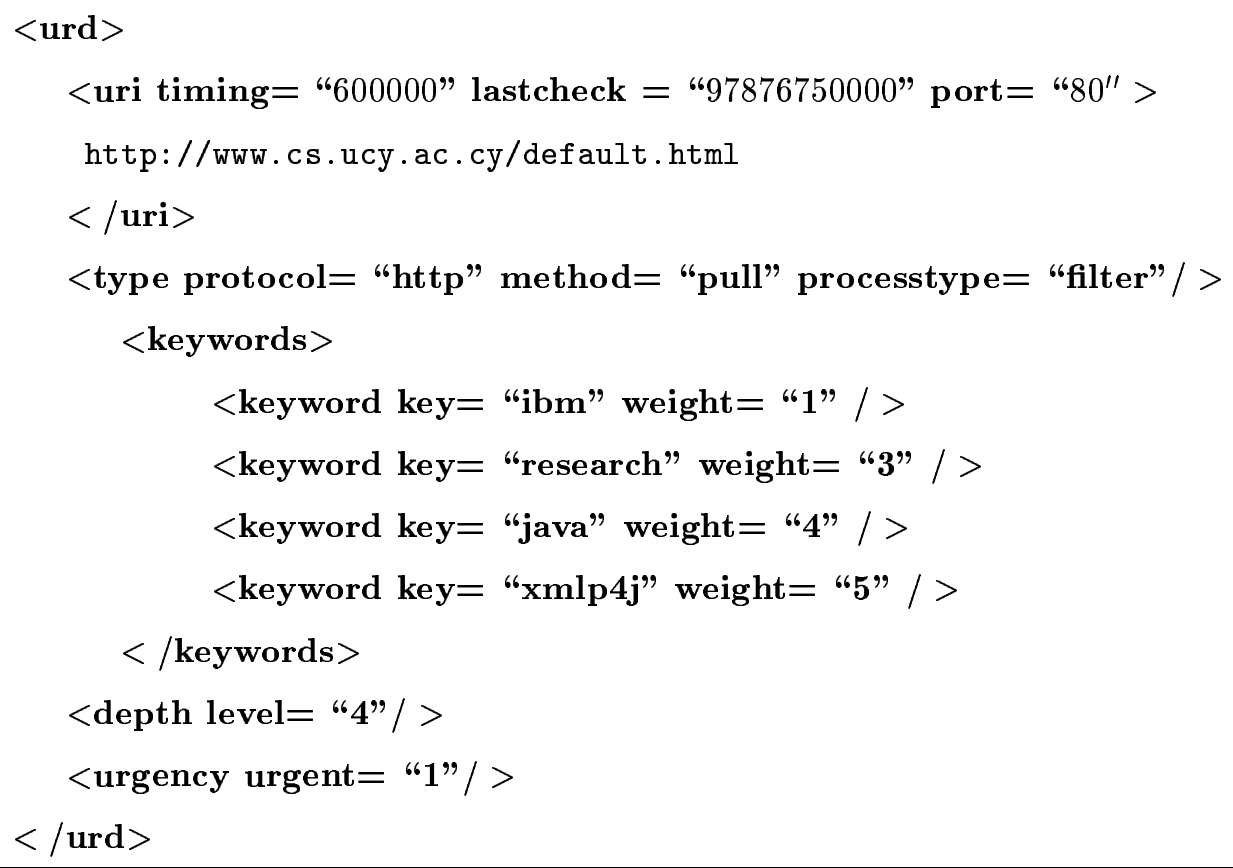

Table 1: A typical URD instance.

in Table 1.

The URD database (URD-DB) is populated by the Service Manager of eRACE. URDDB is a single XML-encoded document managed by $P D O M$, a thread-safe, persistent DOM data manager [26]. XML documents are parsed by PDOM and stored in Java serialized binary form on secondary storage, organized in pages, each containing 128 DOM nodes of variable length. The parsed document is accessible to DOM operations directly, without re-parsing. PDOM nodes accessed by a DOM operation are loaded into a main memory cache. PDOM supports main-memory caching of XML nodes, enabling fast searches in the DOM tree.

Access to URD-DB's contents is provided through the data manager of $P D O M$, which issues XQL queries (eXtensible Query Language) to a GMD-IPSI XQL engine [26, 34]. This engine is a Java-based storage and query application, which handles large XML documents and incorporates two key mechanisms: a persistent implementation of W3C-DOM Document objects [1], and a full implementation of the XQL query language.

The content produced by a URD execution is stored in a software cache of the corresponding Agent-proxy and filtered by the Annotation Engine of eRACE according to processing directives defined in that URD. Meta-information produced by the filtering pro- 


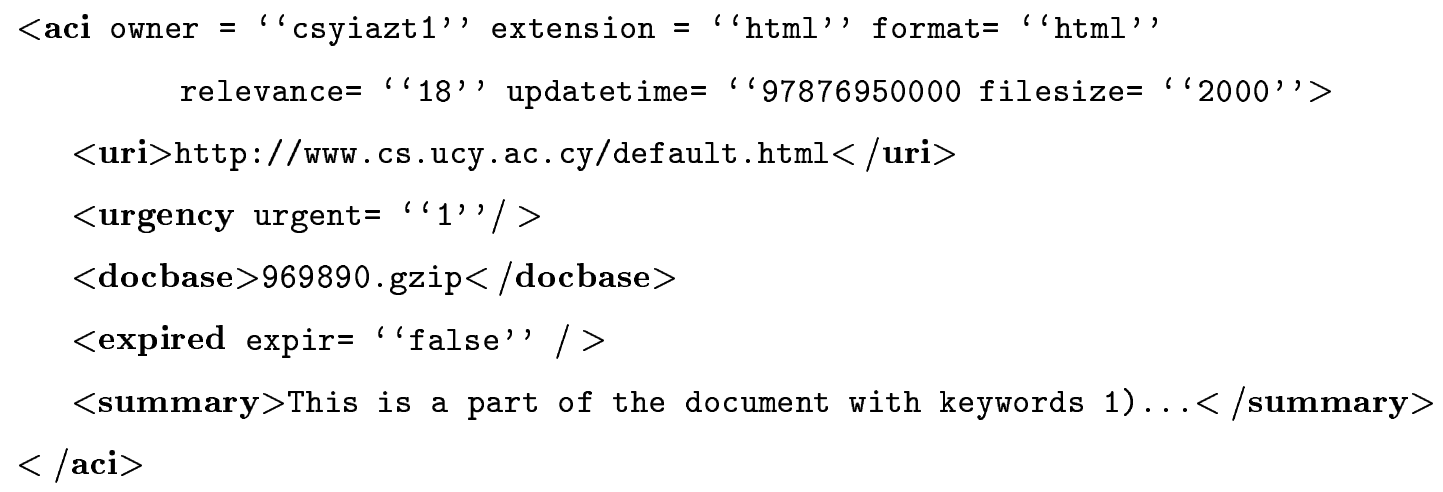

Table 2: ACI snippet.

cess is encoded as an XML data-structure named Annotation Cache Information (ACI) and cached separately. ACI's are used by Content-Distribution Agents for information dissemination to end-users. ACI is an extensible data structure that encapsulates information about the Web source that corresponds to the ACI, the potential user-recipient(s) of the "alert" that will be generated by eRACE's Content Distribution Agents according to the ACI, a pointer to the cached content, a description of the content (format, file size, extension), a classification of this content according to its urgency and/or expiration time, and a classification of the document's relevance with respect to the semantic interests of its potential recipient(s). The XML description of the ACI's is extendible and therefore we can easily include additional information in it without having to change the architecture of WebRACE. ACI's are stored in an XML-ACI PDOM database. An example of a typical ACI snippet is given in Table 2 .

Agent-Proxies implement a number of optimizations such as coalescing different URD requests that target the same information source. Furthermore, Agent-Proxies implement expiration policies that differ from the expiration policies of information sources on Internet. eRACE maintains and manages multiple versions of the content published on some information source, every time this content is of interest to some eRACE profile, and for as long as the resulting information has not been retrieved by interested end-users. This approach makes it necessary to manage obsolete information stored in eRACE caches explicitly. This task is carried out by a Garbage Collector module (see Figure 1). 


\section{WebRACE Design and Implementation Challenges}

WebRACE is the Agent-Proxy that deals with information sources on the WWW and accessible through the HTTP protocols (HTTP/1.0, HTTP/1.1). Other proxies have the same general architecture with WebRACE, differing only in the implementation of their protocol-specific proxy engines.

WebRACE is comprised of three basic components, the Mini-crawler, the Object Cache, and the Annotation Engine. These components operate independently and asynchronously (see Figure 2). They can be distributed to different computing nodes, execute in different Java heap spaces, and communicate through permanent socket links; through these sockets, the Mini-crawler notifies the Annotation Engine every time it fetches and caches a new page in the Object Cache. The Annotation Engine can then process the fetched page asynchronously, according to pre-registered user profiles or other criteria.

In the development of WebRACE we address a number of challenges: First is the design and implementation of a user-driven crawler. Typical crawlers employed by major search engines such as Google [11], start their crawls from a carefully chosen fixed set of "seed" URL's. In contrast, the Mini-crawler of WebRACE receives continuously crawling directives which emanate from a queue of standing eRACE requests (see Figure 2). These requests change dynamically with shifting eRACE-user interests, updates in the base of registered users, changes in the set of monitored resources, etc.

Second, is the design of a crawler that monitors Web-sites exhibiting frequent updates of their content. WebRACE should follow and capture these updates so that interested users are notified by eRACE accordingly. Consequently, WebRACE is expected to crawl and index parts of the Web under short-term time constraints and keep multiple versions of the same Web-page in its store, until all interested users receive the corresponding alerts.

Similarly to personal and site-specific crawlers like SPHINX [32] and NetAttache Pro [27], WebRACE is customized and targets specific Web-sites. These features, however, must be sustained in the presence of a large and increasing user base, with varying interests and different service-level requirements. In this context, WebRACE must be scalable, sustaining high-performance and short turn-around times when serving many users and crawling a large portion of the Web. To this end, it should avoid duplication of effort and combine similar requests when serving similar user profiles. Furthermore, it should provide built- 


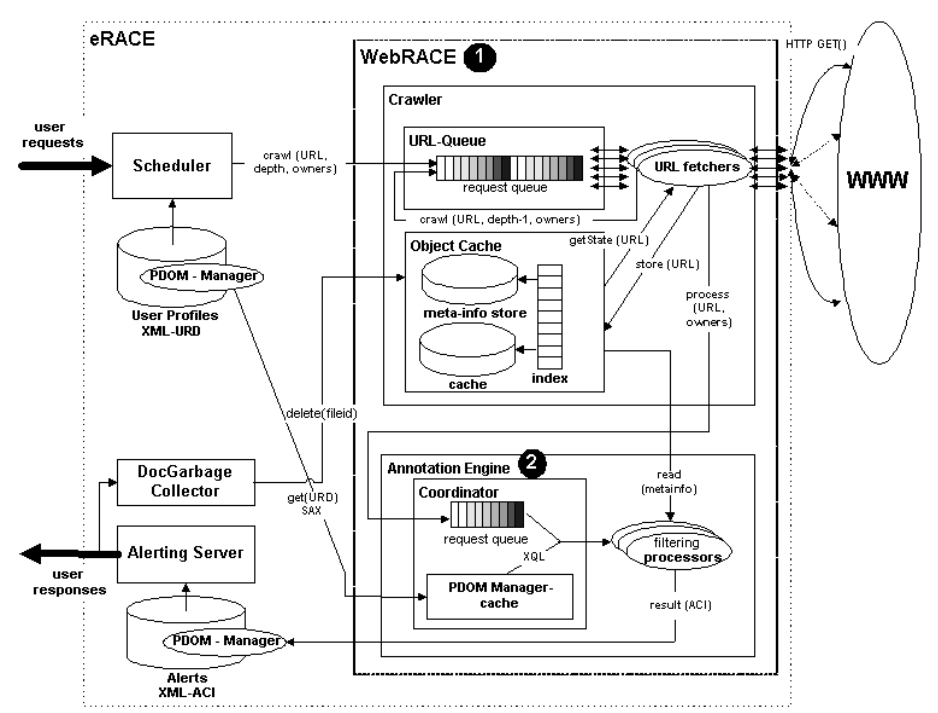

Figure 2: WebRACE System Architecture.

in support for QoS policies involving multiple service-levels and service-level guarantees. Consequently, the scheduling and performance requirements of WebRACE crawling and filtering face very different constraints than systems like Google [11], Mercator [24], SPHINX [32] or NetAttache Pro [27].

Finally, WebRACE is implemented entirely in Java v.1.3. Extensive performance and memory debugging with the OptimizeIt profiler [41], however, identified a number of performance problems arising because of Java core classes (excessive allocation of new objects causing heap-space overflows and performance degradation. Consequently, we developed our own data-structures that use a bounded amount of heap-space regardless of the crawl size, and maintain part of their data on disk. Furthermore, we re-wrote some of the missioncritical Java classes, streamlining very frequent operations. More information on implementation details can be found in [47, 48].

\section{The Mini-crawler of WebRACE}

A crawler is a program that traverses the hypertext structure of the Web automatically, starting from an initial hyper-document and recursively retrieving all documents accessible from that document. Web crawlers are also referred to as robots, wanderers, or spiders. Typically, a crawler executes a basic algorithm that takes a list of "seed" URL's as its 
input, and repeatedly executes the following steps [24]: It initializes the crawling engine with the list of seed URL's and pops a URL out of the URL list. Then, it determines the IP address of the chosen URL's host name, opens a socket connection to the corresponding server, asks for the particular document, parses the HTTP response header and decides if this particular document should be downloaded. If this is so, the crawler downloads the corresponding document and extracts the links contained in it; otherwise, it proceeds to the next URL. The crawler ensures that each extracted link corresponds to a valid and absolute URL, invoking a URL-normalizer to "de-relativize" it, if necessary. Then, the normalized URL is appended to the list of URL's scheduled for download, provided this URL has not been fetched earlier.

In contrast to typical crawlers [32, 24], WebRACE refreshes continuously its URL-seed list from requests posted by the eRACE Request Scheduler. These requests have the following format:

\section{[Link, ParentLink, Depth, \{owners\}]}

Link is the URL address of the Web resource sought, ParentLink is the URL of the page that contained Link, Depth defines how deep the crawler should "dig" starting from the page defined by Link, and \{owners\} contains the list of eRACE users potentially interested in the page that will be downloaded.

The Mini-crawler is configurable through configuration files and can be adapted to specific crawl tasks and benchmarks. The crawling algorithm described in the previous section requires a number of components, which are listed and described in detail below:

- The URLQueue for storing links that remain to be downloaded.

- The URLFetcher that uses HTTP to download documents from the Web. The URLFetcher contains also a URL extractor and normalizer that extracts links from a document and ensures that the extracted links are valid and absolute URL's.

- The Object Cache, which stores and indexes downloaded documents, and ensures that no duplicate documents are maintained in cache. The Object Cache, however, can maintain multiple versions of the same URL, if its contents have changed with time. 


\subsection{The URLQueue}

The URLQueue is an implementation of the SafeQueue data structure that we designed and implemented to achieve the efficient and robust operation of WebRACE and to overcome problems of the java.util.LinkList component of Java [21]. SafeQueue is a circular array of QueueNode objects with its own memory-management mechanism, enabling the re-use of objects and minimizes garbage-collection overhead. Moreover, SafeQueue provides support for persistence, overflow control, disk caching, multi-threaded access, and fast indexing to avoid the insertion of duplicate QueueNode entries [47, 48].

URLQueue is a SafeQueue subclass comprised of $U R L Q u e u e N o d e$ 's, i.e., Java objects that represent requests coming from the Request Scheduler of eRACE. During the server's initialization. The length of the URLQueue is determined during WebRACE initialization from its configuration files. At initialization time, WebRACE allocates the heap-space required to store all the nodes of the queue. This approach is chosen instead of allocating Queue Nodes on demand for memory efficiency and performance. In our experiments, we configured the URLQueue size to two million nodes, i.e., two million URL's. This number corresponds to approximately $27 M B$ of heap space. A larger URLQueue can be employed, however, at the expense of heap size available for other components of WebRACE.

\subsection{The URLFetcher}

The URLFetcher is a multithreaded WebRACE that fetches documents from the Web using the HTTP/1.0 and HTTP/1.1 protocols. URLFetcher threads retrieve grabbing pending requests from the URLQueue, conducting synchronous I/O to download WWW content, and overlapping I/O with computation. In the current version of WebRACE, resource management and thread scheduling is left to Java's runtime system and the underlying operating system. The number of available URLFetcher threads, however, can be configured during the initialization of the WebRACE-server.

In addition to handling HTTP connections, the URLFetcher processes downloaded documents. To this end, it invokes its URLExtractor and normalizer sub-component. The URLExtractor extracts links (URL's) out of a page, disregards URL's pointing to uninteresting resources, normalizes the URL's so that they are valid and absolute and, finally, adds these links to the URLQueue. As shown in Figure 3, the URLExtractor and normalizer 


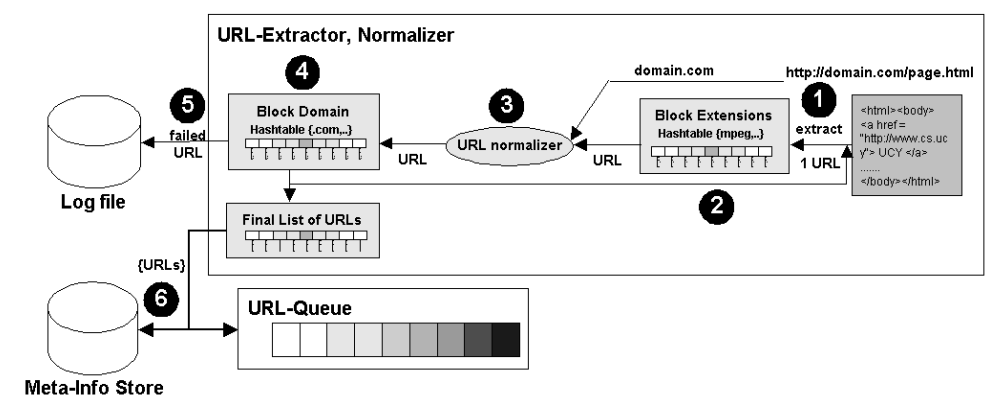

Figure 3: URL Extractor Architecture

works as a 6 -step pipe within the URLFetcher.

\section{The Object Cache}

The Object Cache is the component responsible for managing documents cached in secondary storage. It is used for storing downloaded documents that will be retrieved later for processing, annotation and subsequent dissemination to eRACE users. The Object Cache, moreover, caches the crawling state in order to coalesce similar crawling requests and to accelerate the re-crawling of WWW resources that have not changed since their last crawl.

The Object Cache is comprised of an Index, a Meta-Info Store and an Object Store (see Figure 2). The Index resides in main memory and indexes documents stored on disk; it is implemented as a java.util. HashTable, which contains URL's that have been fetched and stored in WebRACE. That way, URLFetcher's can check if a page has been re-fetched, before deciding whether to download its contents from the Web. The Meta-Info Store collects and maintains meta-information for cached documents. Finally, the Object Store is a directory in secondary storage that contains a compressed version of downloaded resources.

The Meta-Info Store maintains a meta-information file for each Web document stored in the Object Cache. Furthermore, a key for each meta-info file is kept with the Index of the Object Cache to allow for fast look-ups. The contents of a meta-info file are encoded in XML and include: (i) The URL address of the corresponding document; (ii) The IP address of its origin Web server; (iii) The document size in KiloBytes; (iv) The Last-Modified field returned by the HTTP protocol during download; (v) The HTTP response header, and all extracted and normalized links contained in this document. An example of a meta-info file is given in Table 3. Normally, a URLFetcher executes the following algorithm to download 


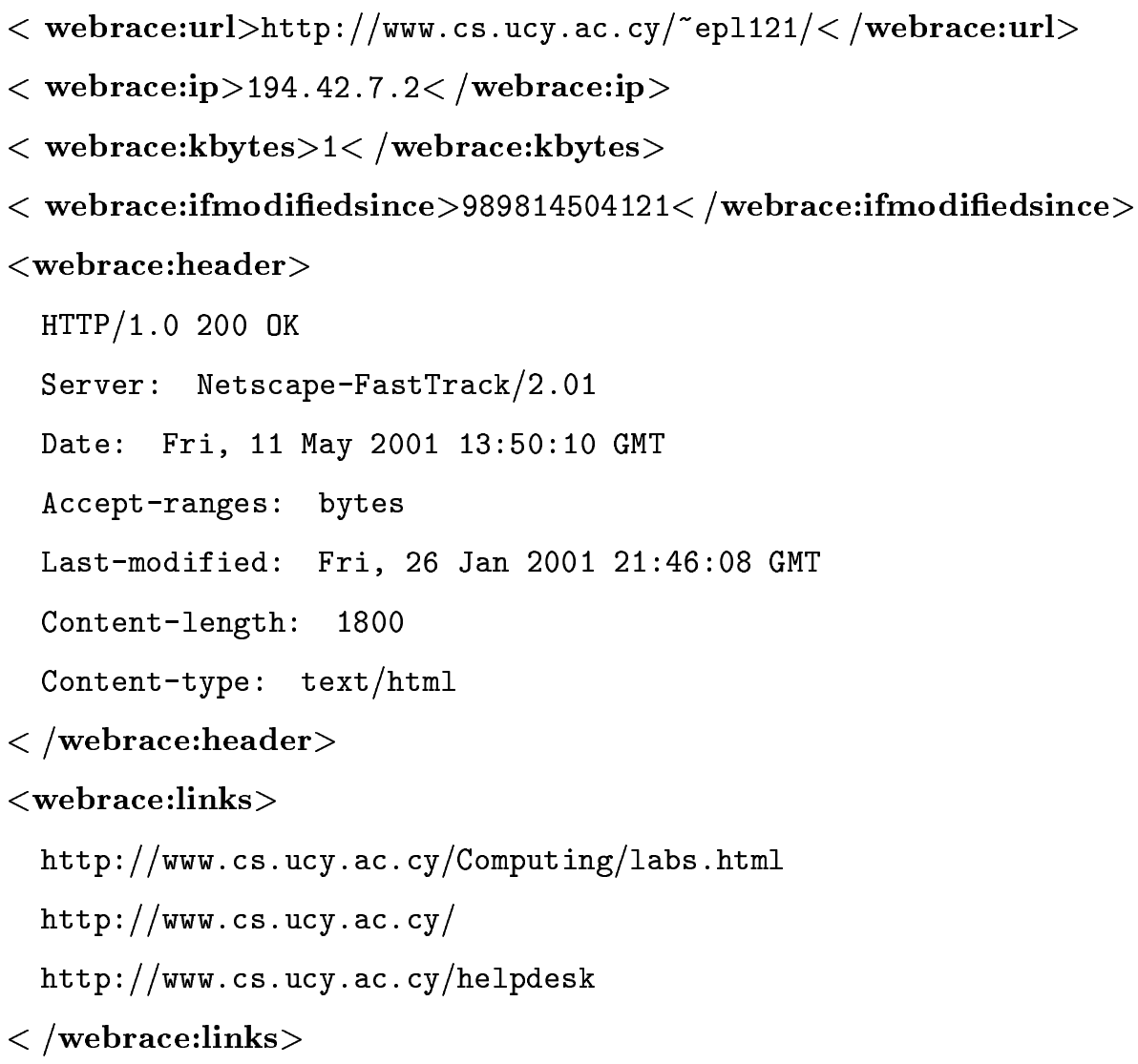

Table 3: Example of meta-information file.

a Web page:

1. Retrieve a QueueNode from the URLQueue and extract its URL.

2. Retrieve the URL and analyze the HTTP-header of the response message. If the host server contains the message "200 Ok," proceed to the next step. Otherwise, continue with the next QueueNode.

3. Download the body of the document and store it in main memory.

4. Extract and normalize all links contained in the downloaded document.

5. Compress and save the document in the Object Cache.

6. Save a generated meta-info file in the Meta-Info Store.

7. Add the key (hashCode) of the fetched URL to the Index of the Object Cache. 
8. Notify the Annotation Engine that a new document has been fetched and stored in the Object Cache.

9. Add all extracted URL's to the URLQueue.

To avoid the overhead of the repeated downloading and analysis of documents that have not changed, we alter the above algorithm and use the Meta-Info Store to decide whether to download a document that is already cached in WebRACE. More specifically, we change the second and third steps of the above crawling algorithm as follows:

2. Access the Index of the Object Cache and check if the URL retrieved from the URLQueue corresponds to a document fetched earlier and cached in WebRACE.

3. If the document is not in the Cache, download it and proceed to step 4. Otherwise:

- Load its meta-info file and extract the HTTP Last-Modified time-stamp assigned by the origin server. Open a socket connection to the origin server and request the document using a conditional HTTP GET command (if-modified-then), with the extracted time-stamp as its parameter.

- If the origin server returns a "304 (not modified)" response and no messagebody, terminate the fetching of this particular resource, extract the document links from its meta-info file, and proceed to step 8.

- Otherwise, download the body of the document, store it in main memory and proceed to step 4.

If a cached document has not been changed during a re-crawl, the URLFetcher proceeds with crawling the document's outgoing links, which are stored in the Meta-Info Store, and which may have changed.

\section{The Annotation Engine (AE)}

The Annotation Engine processes documents that have been downloaded and cached in the Object Cache of WebRACE. Its purpose is to "classify" collected content according to userinterests described in eRACE profiles. The meta-information produced by the processing 


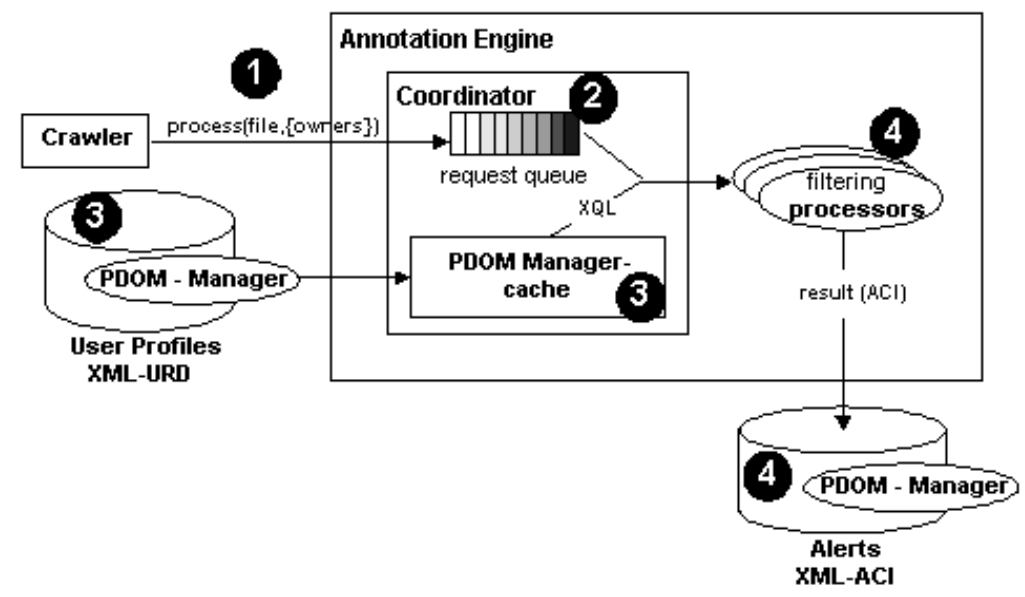

Figure 4: WebRACE Annotation Engine.

of the Annotation Engine is stored as eRACE annotations (ACI's) linked to the cached content. Pages that are not relevant to any user-profile are dropped from the cache.

Personalized annotation engines are not used in typical Search Engines [11], which employ general-purpose indices instead. To avoid the overhead of incorporating a generic look-up index in WebRACE that will be updated dynamically as resources are downloaded from the Web, we designed the AE so that it processes downloaded pages "on the fly." Therefore, each time the Annotation Engine receives a ' process(file, \{users\})' request through established socket connections with the Mini-crawler, it inserts the request in the Coordinator, which is a SafeQueue data structure (see Figure 4). Multiple Filtering Processors remove requests from the Coordinator and process them according to the Unified Resource Descriptions (URD's) of eRACE users contained in the request. Currently, the annotation engine implements a pattern-matching algorithm looking for weighted keywords that are included in the user-profiles, similar to that of [44].

Filtering Processor (FP) is the component responsible for evaluating if a document matches the interests of a particular eRACE-user, and for generating an ACI out of a crawled page (see Figure 5). The Filtering Processor works as a pipe of filters: At step 1, FP loads and decompresses the appropriate file from the Object Cache of WebRACE. At step 2, it removes all links contained in the document and proceeds to step 3, where all special HTML characters are also removed. At step 4, any remaining text is added to a Keyword HashTable. Finally, at step 5, a pattern-matching mechanism loads sequentially 


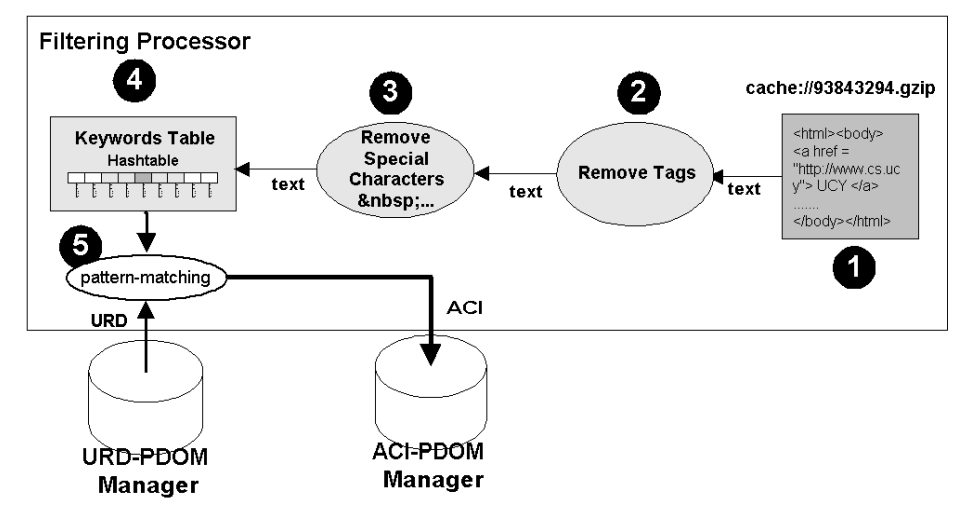

Figure 5: The Filtering Processor.

all the required URD elements from the URD-PDOM and generates ACI meta-information, which is stored in the ACI-PDOM (step 6). This pipe requires an average of 200 msecs to calculate the ACI for a $70 K B$ Web page, with 3 potential recipients.

In our experiments, we have configured the SafeQueue size of the Annotation Engine to 1000 nodes, which proved to be adequately large in experiments conducted with 10 Filtering-processor threads and 100 URLFetcher threads.

\section{Experiments and Performance Assessment}

To evaluate the performance of WebRACE we ran a number of tests and extracted measurements of performance-critical components. In particular, we investigated the benefits of caching the crawling state, the overall performance of the crawler, and the effects that multithreading has on URLFetcher performance. For our experiments, we crawled three classes of Web sites: the first class includes servers that provide content which does not change very frequently (remote University sites in the U.S.); the second class consists of popular news-sites, search-engine sites and portals (cnn.com, yahoo.com, msn.com, etc.); the third class consists of Web servers residing in our departmental network.

\subsection{Overall Performance and Benefits from Caching Crawling State}

To assess the performance improvement achieved caching crawling state in the Meta-Info Store, we conducted experiments with the first two classes of Web sites. For these experiments we configured WebRACE to use 150 concurrent URLFetchers and ran it on our 
-. Crawling

- .- Re-Crawling after 1 hour with Meta-Info Store

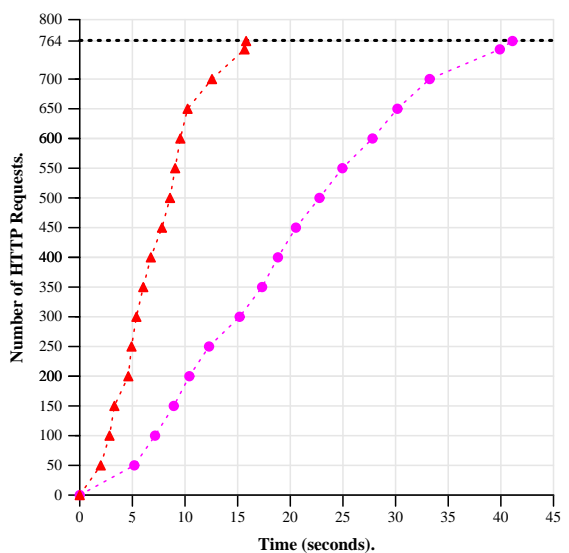

[Crawl vs. Re-crawl, 25 US Universities (2 levels)]
... Crawling 10 Portals (2 levels each)

- - Re-Crawling after 10 minutes with Meta-Info Store.

-. Re-Crawling after 1 hour with Meta-Info Store.

-.. Re-Crawling after 1 hour without Meta-Info Store.

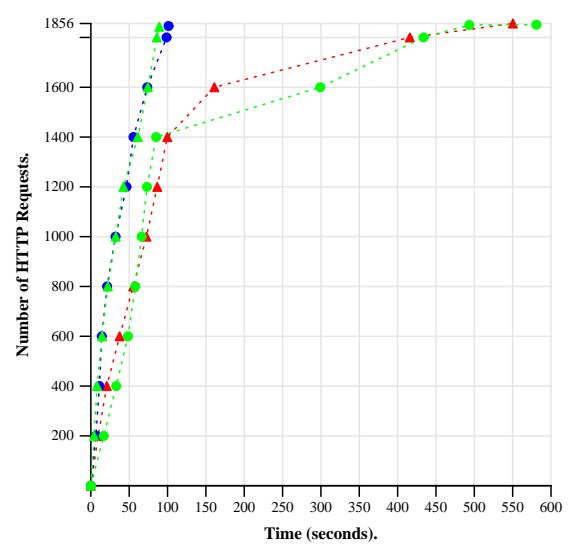

[Crawl .vs ReCrawl 10 Frequently Changed Portals (2 levels)]

Figure 6: Crawling vs. re-crawling in WebRACE.

dual-processor Sun Enterprise E250 Server, with the Annotation Processor running concurrently with 10 threads on a Sparc 5 .

The diagram of Figure 6 (left) presents the progress of the crawl and re-crawl operations for the first class of sites. The time interval between the crawl and the subsequent re-crawl was one hour; within that hour the crawled documents had not changed at all. The delay observed for the re-crawl operation is attributed to the HTTP "if-modified-since" validation messages and the overhead of the Object Cache. As we can see from this diagram, the employment of the Meta-Info Store results to an almost three-fold improvement in the crawling performance. Moreover, it reduces substantially the network traffic and the Webservers' load generated because of the crawl.

The diagram of Figure 6 (right) presents our measurements from the crawl and re-crawl operations for the second class of sites. Here, almost $10 \%$ of the 993 downloaded documents change between subsequent re-crawls. From this diagram we can easily see the performance advantage gained by using the Meta-Info Store to cache crawling meta-information: from a throughput of 3.37 requests/sec of the first crawl, we achieve throughput of 18.31 and 20.77 requests/sec for the two subsequent re-crawls, respectively.

It should be noted that within the first 100 msecs of all these crawling experiments, crawling and re-crawling exhibit practically the same performance behavior. This is attributed to the fact that most of the crawled portals reply to our HTTP GET requests with 


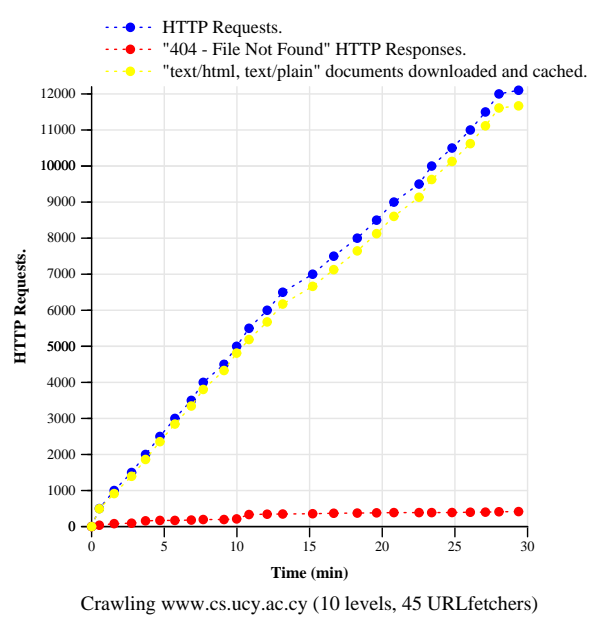

Figure 7: Performance of a longer crawl.

"301 (Moved Permanently)" responses, and re-direct our crawler to other URL's. In these cases, the crawler terminates the connection and schedules immediately a new HTTP GET operation to fetch the requested documents from the re-directed address.

Finally, in Figure 7, we present measurements from a longer crawl that took 29.38 mins to complete and produced 11669 documents. This crawl was conducted on our (slow) departmental Web servers.

\subsection{URLFetcher Performance}

To evaluate the overall performance of the URLFetcher, we ran a number of experiments launching many concurrent fetchers that try to establish TCP connections and fetch documents from Web servers located on our 100Mbits LAN. Each URLFetcher pre-allocates all of its required resources before the benchmark start-up. The benchmarks ran on a $360 \mathrm{MHz}$ UltraSPARC-IIi, with 128MB RAM and Solaris 5.7.

As we can see from Figure 8, the throughput increases with the number of concurrent URLFetchers, until a peak $\mathrm{P}$ is reached. After that point, throughput drops substantially, because of the synchronization overhead incurred by the large number of concurrent threads. This crawling process took a very short time ( 3 minutes with only one thread), which is actually the reason why the peak value $\mathrm{P}$ is 40 . In this case, URLQueue empties very fast, limiting the utilization of URLFetcher's near the benchmark's end. Running the same benchmark for a lengthy crawl we observed that 100 concurrent URLFetcher's achieve 


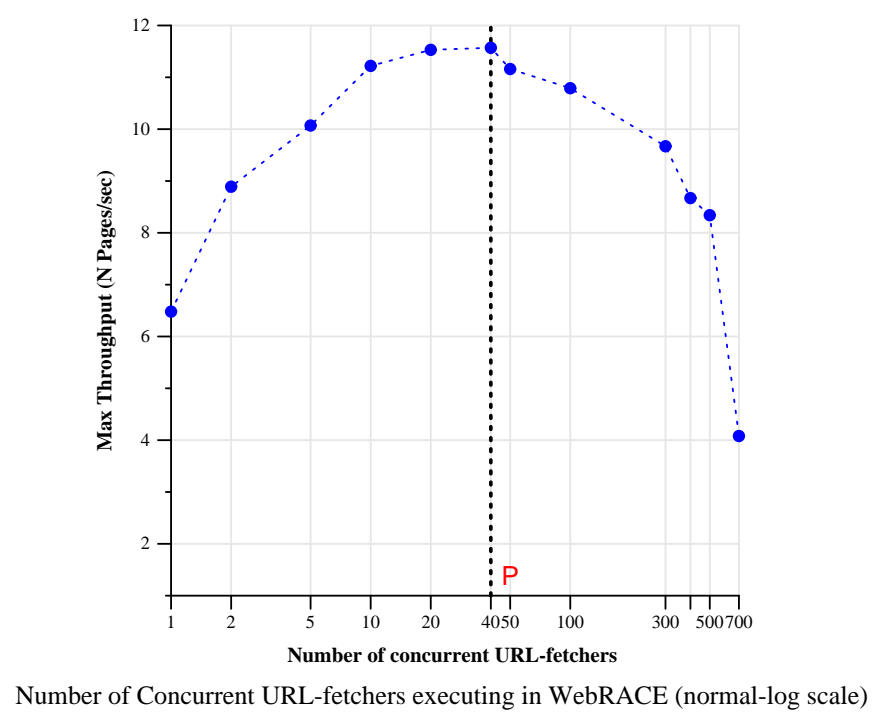

Figure 8: URLFetcher throughput degradation.

optimal crawling throughput.

\subsection{Distributed Mini-Crawler Execution}

In order to speed-up the performance of WebRACE, we sought to parallelize the MiniCrawler by running multiple multithreaded URLFetcher's on a network of workstations, keeping the number of threads at each workstation optimal. To this end, we employed the Java-based Distributed Data Structure component, which was developed at UC/Berkeley [22]. The DDS component distributes and replicates the contents of a data-structure across a cluster or network of workstations, providing the programmer with a conventional, singlesite, in-memory interface to the data structure [22]. Coherence between distributed replicas is established through an implementation of a two-phase commit protocol, which is transparent to the programmer of services on top of DDS.

When using the DDS layer and API, we kept the core of WebRACE intact and added-on a module that could handle the distribution of the Mini-Crawler. To this end, we ran multiple URLFetchers on different machines and used the Distributed Hashtable implementation of DDS [22] to index the documents gathered by the URLFetchers, allowing the crawlers to know the content of the Meta-Info store. The total time spent to incorporate the DDS in WebRACE and make it distributed was less that 3 hours.

The topology of the distributed Mini-Crawler is presented in Figure 9. We employed 


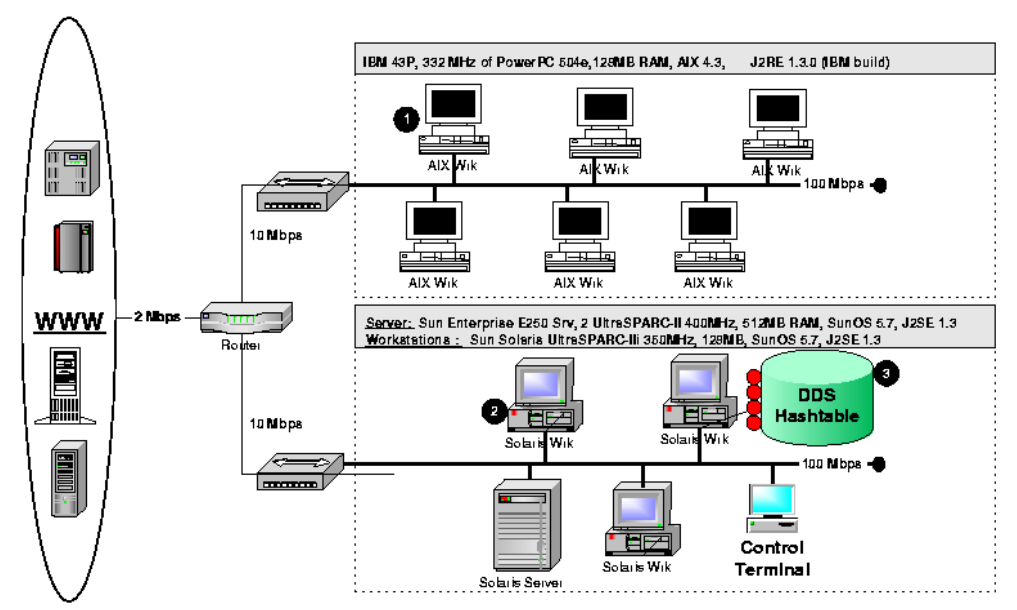

Figure 9: WebRACE Distributed Crawler Network Topology.

eight crawling nodes, one DDS indexing node, and one control terminal for monitoring and control. The crawling nodes are IBM 43P workstations running AIX, SPARC workstations running Solaris, and a dual processor Sun Enterprise E250 server, all connected on our 10/100 Mbps Local Area Network.

To assess the performance improvement provided by the distribution of WebRACE, we conducted an experiment crawling our first class of servers (University sites) in a depth of 3 levels. To this end, we configured each crawling node to use only 45 concurrent URLFetchers. Measurements are presented in Figure 10, which shows that the performance of the distributed WebRACE scales linearly with the number of crawling nodes. It is interesting to note that for 45 concurrent URLFetcher threads, the performance of the distributed WebRACE with one crawling node is better than that of the "standalone" WebRACE. This is attributed to the poor performance of the synchronized java.util. HashTable used in the latter case. Finally, different crawling nodes display a different crawling throughput: on the average, the E250 dual processor server was three times faster than the SPARC-stations, and almost ten times faster than the AIX machines.

\section{Related Work}

Research and development on intermediary software for Internet has produced a variety of systems, ranging from proxy servers [7, 42], subscription-based notification and information dissemination services $[44,17,3,36,15,14]$, tools that support mobile Web access $[25$, 


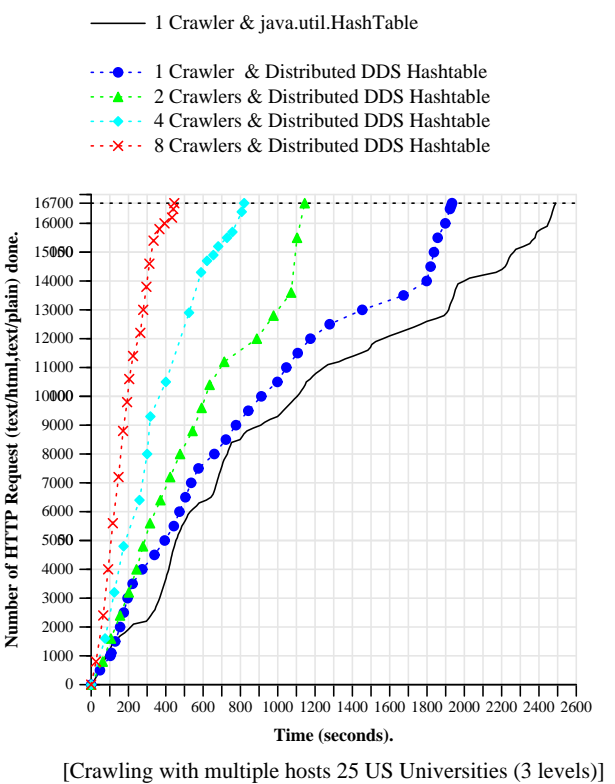

Figure 10: WebRACE Distributed Crawler Performace.

19], and more generic, programmable infrastructures that can be used to build ubiquitous services $[4,9,20,23,18]$. These systems fall under the general term of "middleware," as they provide a "reusable and expandable set of services and functions, commonly needed by many applications to function well in a networked environment" [2]. Here, we describe some of the more recent and ambitious examples of intermediary infrastructures.

A programmable framework for building various intermediary services is the Web Browser Intelligence or WeB Intermediaries (WBI) by IBM Almaden [5, 4, 31]. WBI is a programmable proxy server designed for the development and deployment of intermediary applications. The design of WBI is based on the notion of "information streams" that convey data from information providers (e.g., a Web server) to information consumers (e.g., a Web browser), and can be classified into unidirectional and bidirectional messages or transaction streams [6]. WBI has been used to develop a number of applications such as a managerrepository for cookies and a Web-browsing service for mobile devices [4]. WBI plugins can be installed both on a client machine and on any other networked machine, possibly near an origin server. Multiple client-side and server-side WBI intermediaries can cooperate to establish one WBI service.

Building mobile services from proxy components is the main goal of the iMobile project of AT\&T Research [38]. The iMobile proxy maintains user and device profiles, accesses 
and processes Internet resources on behalf of the user, keeps track of user interaction and performs content transformations according to device and user profiles. The architecture of iMobile is established upon the infrastructure of iProxy, a programmable proxy server designed to host agents and personalized services developed in Java [39]. iMobile consists of three main abstractions: devlets, infolets and applets. A devlet is an agent-abstraction for supporting the provision of iMobile services to different types of mobile devices connected through various access networks. A devlet-instance communicates with a device-specific "driver" that is either co-located in the iProxy server or resides at a remote mobile support station. The infolet abstraction provides a common way of expressing the interaction between the iMobile server and various information sources or information spaces (in iMobile terminology) at a level higher than the HTTP protocol and the URI specification. Different sources export different interfaces to the outside world: JDBC and ODBC for corporate databases, the X10 protocol for home networks, IMAP for email servers, etc. Finally, an applet is a module that processes and aggregates content retrieved by different sources and relays results to various destination devices. At the core of an iMobile server resides the "let engine," which registers all devlets, infolets and applets, receives commands from devlets, forwards them to the right infolet or applet, transcodes the result to an appropriate terminal-device format and forwards it to the terminal device via the proper devlet.

A broader approach that seeks to develop a robust infrastructure and programming platform for Internet-scale systems and services in Java comes from the Ninja project at UC/Berkeley [23]. The architecture of Ninja consists of bases, active proxies, units and paths. Bases are scalable platforms designed to host Internet services. They consist of a programming model and I/O substrate designed to provide high-concurrency, robustness, and transparent distribution of data to cluster-nodes [22]. Moreover, they include a cluster-based execution environment (vSpace) that provides facilities for service component replication, load-balancing and fault-tolerance [23]. The programming model of Ninja consists of four design patterns that service programmers can use to compose different stages of a single service: wrap, pipeline, combine and replicate [23]. Active proxies are fine-grain intermediaries providing transformational support between Ninja services and terminal devices. Active proxies perform data distillation, protocol adaptation, caching, encryption, etc. Examples of active proxies include wireless base-stations, network gateways, firewalls, 
and caching proxies. In Ninja terminology, a path is a flow of typed data through multiple proxies across a wide-area network; each proxy performs transformational operations to adapt the data into a form acceptable by the next service or device along the path. Similarly to WBI plugins, Ninja-paths can be established dynamically. Finally, units are abstractions for the client devices attached to the Ninja infrastructure, which range from PC's and laptops to mobile devices, sensors and actuators.

In our work, we address the challenge of designing and implementing modular, open, distributed, and scalable intermediary infrastructures, using Java. Our effort differs from other systems in a number of key issues: (i) Our design is established upon a set of XML grammars that define the information and meta-information collected, generated and exchanged between the various modules of our system. The employment of XML makes it easier to decouple information retrieval, storage and processing from content publishing and distribution. Furthermore, it enables us to describe easily new services, terminal devices, and information sources, without altering the basic design of our infrastructure. Finally, it exports the operations "executed" by the engine to the system-programming level, thus making possible the implementation of different scheduling and resource-management policies according to differentiated service-levels provided to individual users or services. (ii) eRACE consists of modules that communicate via messages and events; therefore, it is relatively easy to distribute these modules to different machines, achieve distributed operation and scalable performance. (iii) Instead of making eRACE a totally generic infrastructure, we developed and optimized modules which provide a functionality necessary nowadays in most intermediary systems: a "user-driven" high-performance crawler, an object cache, and a filtering processor. These, performance-critical modules are multithreaded and distributed thanks to the employment of Java and of distributed data structures in Java. (iv) The development of new services on top of eRACE is supported through the definition of new XML profiles inserted at the information architecture of eRACE. Additional programmability is provided by the employment of the mobile-agent programming paradigm, which supports mobility and disconnected operation of end-users as well. (v) Although a large number of papers have been published on Web crawlers [32, 24, 13, 12, 37], and Internet middleware, the issue of incorporating flexible, scalable and user-driven crawlers in middleware infrastructures remains open. This issue is addressed in the context of WebRACE. 


\section{Conclusions and Future Work}

In this paper, we presented WebRACE, a World-Wide Web "agent-proxy" that collects, filters and caches Web documents. WebRACE is designed in the context of eRACE, an extensible Retrieval Annotation Caching Engine. WebRACE consists of a high-performance, distributed Web crawler, a filtering processor, and an object cache, written entirely in Java. In our work, we addressed the challenges arising from the design and development of WebRACE. We described our design and implementation decisions, and various optimizations. Furthermore, we discussed the advantages and disadvantages of using Java to implement the crawler, and presented an evaluation of its performance.

To assess WebRACE's performance and robustness we ran numerous experiments and crawls; several of our crawls lasted for days. Our system worked efficiently and with no failures when crawling local Webs in our LAN and University WAN, and the global Internet. Our experiments showed that our implementation is robust and reliable. The combination of techniques such as, multithreading, caching the crawling state, and the employment of distributed data stuctures in Java, improved the scalability and performance of WebRACE.

Further optimizations will be included in the near future, so as to prevent our crawler from overloading remote Web servers with too many concurrent requests. We also plan to investigate alternative queue designs and different crawling strategies (breadth-first versus depth-first) that have been reported to provide improved crawling efficiency.

\section{References}

[1] Document Object Model (DOM) Level 1 Specification. W3C Recommendation 1, October 1998. http://www.w3.org/TR/REC-DOM-Level-1/.

[2] R. Aiken, M. Carey, B. Carpenter, I. Foster, C. Lynch, J. Mambreti, R. Moore, J. Strasnner, and B. Teitelbaum. Network Policy and Services: A Report of a Workshop on Middleware. Technical Report RFC 2768, IETF, 2000. http://www.ietf.org/rfc/rfc2768.txt.

[3] D. Aksoy, M. Altinel, R. Bose, U. Cetintemel, M.J. Franklin, J. Wang, and S.B. Zdonik. Research in Data Broadcast and Dissemination. In Proceedings of the First Interna- 
tional Conference on Advanced Multimedia Content Processing, AMCP '98, Lecture Notes in Computer Science, pages 194-207. Springer Verlag, 1999.

[4] R. Barrett and P. Maglio. Intermediaries: New Places for Producing and Manipulating Web Content. Computer Networks and ISDN Systems, 30(1-7):509-518, April 1998.

[5] R. Barrett and P. Maglio. Intermediaries: New places for producing and manipulating Web content. In Proceedings of the Seventh International World Wide Web Conference (WWW'), 1998.

[6] R. Barrett and P. Maglio. Intermediaries: An approach to manipulating information streams. IBM Systems Journal, 38(4):629-641, 1999.

[7] C. M. Bowman, P. B. Danzig, D. R. Hardy, U. Manber, and M. F. Schwartz. The Harvest Information Discovery and Access System. In Proceedings of the Second International WWW Conference, 1995.

[8] T. Bray, J. Paoli, and C.M. Sperberg-McQueen. Extensible Markup Language (XML) 1.0. W3C Recommendation. Technical Report REC-xml-19980210, World-Wide Web Consortium, February 1998. http://www.w3.org/TR/.

[9] E. Brewer, R. Katz, E. Amir, H. Balakrishnan, Y. Chawathe, A. Fox, S. Gribble, T. Hodes, G. Nguyen, V. Padmanabhan, M. Stemm, S. Seshan, and T. Henderson. A Network Architecture for Heterogeneous Mobile Computing. IEEE Personal Communications Magazine, 5(5):8-24, October 1998.

[10] E. A. Brewer. Lessons from Giant-Scale Services. Internet Computing, 5(4):46-55, July-August 2001.

[11] S. Brin and L. Page. The Anatomy of a Large-Scale Hypertextual (Web) Search Engine. Computer Networks and ISDN Systems, 30(1-7):107-117, 1998.

[12] S. Chakrabarti, M. van den Berg, and B. Dom. Focused Crawling: A New Approach to Topic-Specific Web Resource Discovery. In 8th World Wide Web Conference, Toronto, May 1999. 
[13] J. Cho, H. Garcia-Molina, and L. Page. Efficient crawling through URL ordering. In Proceedings of the Seventh International WWW Conference, pages 161-172, April 1998.

[14] M. Dikaiakos. FIGI: Using Mobile Agent Technology to Collect Financial Information on Internet. In Workshop on Data Mining in Economics, Marketing and Finance. Machine Learning and Applications. Advanced Course on Artificial Intelligence 1999 (ACAI '99). European Coordinating Committee on Artificial Intelligence and Hellenic Artificial Intelligence Society, July 1999.

[15] M. Dikaiakos and D. Gunopulos. FIGI: The Architecture of an Internet-based Financial Information Gathering Infrastructure. In Proceedings of the International Workshop on Advanced Issues of E-Commerce and Web-based Information Systems, pages 91-94. IEEE-Computer Society, April 1999.

[16] M. Dikaiakos, M. Kyriakou, and G. Samaras. Performance Evaluation of Mobile-Agent Middleware: A Hierarchical Approach. In G. P. Picco, editor, Proceedings of the 5th International Conference on Mobile Agents (MA 2001), volume 2240 of Lecture Notes in Computer Science, pages 244-259. Springer, 2002.

[17] F. Douglis, T. Ball, Y.-F. Chen, and E. Koutsofios. The AT\&T Internet Difference Engine: Tracking and Viewing Changes on the Web. World Wide Web, 1(1):27-44, January 1998.

[18] P. Farjami, C. Gorg, and F. Bell. Advanced Service Provisioning Based on Mobile Agents. Computer Communications, (23):754-760, 2000.

[19] A. Fox, I. Goldberg, S. Gribble, D. Lee, A. Polito, and E. Brewer. Experience with Top Gun Wingman: A Proxy-based Graphical Web Browser for the 3Com PalmPilot. In Proceedings of the IFIP International Conference on Distributed Systems Platforms and Open Distributed Processing (Middleware '98), pages 407-426, 1998.

[20] A. Fox, S.D. Gribble, Y. Chawathe, and E.A. Brewer. Adapting to Network and Client Variation Using Active Proxies: Lessons and Perspectives. IEEE Personal Communications, 5(4):10-19, August 1998. 
[21] J. Gosling, B. Joy, and G. Steele. The Java Language Specification. Addison-Wesley, 1996.

[22] S. Gribble, E. Brewer, J. Hellerstein, and D. Culler. Scalable, Distributed Data Structures for Internet Service Construction. In Proceedings of the Fourth Symposium on Operating Systems Design and Implementation (OSDI 2000), 2000.

[23] S. Gribble, M. Welsh, R. von Behren, E. Brewer, D. Culler, N. Borisov, S. Czerwinski, R. Gummadi, J. Hill, A. Joseph, R.H. Katz, Z.M. Mao, S. Ross, and B. Zhao. The Ninja Architecture for Robust Internet-Scale Systems and Services. To appear in a Special Issue of Computer Networks on Pervasive Computing, 2001.

[24] A. Heydon and M. Najork. Mercator: A Scalable, Extensible Web Crawler. World Wide Web, 2(4):219-229, December 1999.

[25] B.C. Housel, G. Samaras, and D.B. Lindquist. WebExpress: A Client/Intercept Based System for Optimizing Web Browsing. ACM/Baltzer Journal of Mobile Neworking and Applications (MONET), 3:419-431, 1998.

[26] G. Huck, I. Macherius, and P. Fankhauser. PDOM: Lightweight Persistency Support for the Document Object Model. In Proceedings of the 1999 OOPSLA Workshop Java and Databases: Persistence Options. Held on the 14th Annual ACM SIGPLAN Conference on Object-Oriented Programming Systems, Languages, and Applications (OOPSLA '99). ACM, SIGPLAN, November 1999.

[27] Tympani Development Inc. NetAttache Pro. http://www.tympani.com/products/NAPro.html, 2000 .

[28] A. Joshi. On proxy agents, mobility, and web access. Mobile Networks and Applications, $5: 233-241,2000$.

[29] R. Koblick. Concordia. Communications of the ACM, 42(3):96-99, March 1999.

[30] Horizon Systems Laboratory. Mobile Agent Computing. A White Paper. Mitsubishi Electric ITA., January 1998.

[31] P. Maglio and R. Barrett. Intermediaries Personalize Information Streams. Communications of the $A C M, 43(8): 96-101$, August 2000. 
[32] R. Miller and K. Bharat. SPHINX: A Framework for Creating Personal, Site-specific Web Crawlers. In Proceedings of the Seventh International WWW Conference, pages 161-172, April 1998.

[33] D. Milojicic. Internet Technology. IEEE Concurrency, pages 70-81, January-March 2000 .

[34] GMD-IPSI XQL Engine. http://xml.darmstadt.gmd.de/xql/.

[35] M. Perkowitz and O. Etzioni. Towards adaptive Web sites: Conceptual framework and case study. Artificial Intelligence, 118:245-275, 2000.

[36] S.H. Phatak, V. Esakki, B.R. Badrinath, and L. Iftode. Web\&: An Architecture for Non-Interactive Web. Technical Report DCS-TR-405, Department of Computer Science, Rutgers University, December 1999.

[37] S. Raghavan and H. Garcia-Molina. Crawling the Hidden Web. In VLDB 2001: 27th International Conference on Very Large Data Bases, September 2001. To appear.

[38] H. Rao, Y. Chen, D. Chang, and M. Chen. iMobile: A Proxy-based Platform for Mobile Services. In The First ACM Workshop on Wireless Mobile Internet (WMI 2001), 2001.

[39] H. Rao, Y. Chen, and M. Chen. A Proxy-based Web Archiving Service. In Middleware Symposium, 2000.

[40] G. Samaras, M. Dikaiakos, C. Spyrou, and A. Liverdos. Mobile Agent Platforms for Web-Databases: A Qualitative and Quantitative Assessment. In Proceedings of the Joint Symposium ASA/MA '99. First International Symposium on Agent Systems and Applications (ASA '99). Third International Symposium on Mobile Agents (MA '99), pages 50-64. IEEE-Computer Society, October 1999.

[41] VMGEAR. OptimizeIt!: The Java Ultimate Performance Profiler. http://www.vmgear.com/.

[42] D. Wessels and K. Claffy. Evolution of the NLANR Cache Hierarchy: Global Configuration Challenges. Technical report, NLANR, October 1996. http://www.nlanr.net/Papers/Cache96/. 
[43] T. W. Yan and H. Garcia-Molina. SIFT - A Tool for Wide-Area Information Dissemination. In Proceedings of the 1995 USENIX Technical Conference, pages 177-186, 1995.

[44] T. W. Yan and H. Garcia-Molina. SIFT - A Tool for Wide-Area Information Dissemination. In Proceedings of the 1995 USENIX Technical Conference, pages 177-186, 1995.

[45] N. J. Yeager and R. E. McGrath. Web Server Technology. Morgan Kaufmann, 1996.

[46] D. Zeinalipour-Yazti. eRACE: an eXtensible Retrieval, Annotation and Caching Engine, June 2000. B.Sc. Thesis. In Greek.

[47] D. Zeinalipour-Yazti and M. Dikaiakos. High-Performance Crawling and Filtering in Java. In Proceedings of the Eighth Panhellenic Conference on Informatics, volume 2, pages 377-386, November 2001.

[48] D. Zeinalipour-Yazti and M. Dikaiakos. High-Performance Crawling and Filtering in Java. Technical Report TR-01-3, Department of Computer Science, University of Cyprus, June 2001. 